\title{
Strong convergence of a modified Halpern-type iteration for asymptotically quasi- $\phi$-nonexpansive mappings
}

\author{
Chang-Qun Wu and Yan Hao
}

\begin{abstract}
In this paper, the problem of modifying Halpern iteration for approximating a common fixed point of a family of asymptotically quasi$\phi$-nonexpansive mappings is considered. Strong convergence theorems are established in a uniformly smooth and strictly convex Banach space which also enjoys the Kadec-Klee property. The results presented in this paper mainly improve the corresponding results announced in [Y.J. Cho, X. Qin, S.M. Kang, Strong convergence of the modified Halperntype iterative algorithms in Banach spaces, An. Stiint. Univ. Ovidius Constanta Ser. Mat. 17 (2009) 51-68].
\end{abstract}

\section{Introduction-Preliminaries}

Let $E$ be a Banach space with the dual $E^{*}$. We denote by $J$ the normalized duality mapping from $E$ to $2^{E^{*}}$ defined by

$$
J x=\left\{f^{*} \in E^{*}:\left\langle x, f^{*}\right\rangle=\|x\|^{2}=\left\|f^{*}\right\|^{2}\right\},
$$

where $\langle\cdot, \cdot\rangle$ denotes the generalized duality pairing.

Key Words: asymptotically quasi- $\phi$-nonexpansive mapping, asymptotically nonexpansive mapping, relatively nonexpansive mapping, generalized projection.

2010 Mathematics Subject Classification: Primary 47H09; Secondary 47H10.

Received: September, 2011.

Revised: January, 2012.

Accepted: April, 2012. 
A Banach space $E$ is said to be strictly convex if $\left\|\frac{x+y}{2}\right\|<1$ for all $x, y \in$ $E$ with $\|x\|=\|y\|=1$ and $x \neq y$. It is said to be uniformly convex if $\lim _{n \rightarrow \infty}\left\|x_{n}-y_{n}\right\|=0$ for any two sequences $\left\{x_{n}\right\}$ and $\left\{y_{n}\right\}$ in $E$ such that $\left\|x_{n}\right\|=\left\|y_{n}\right\|=1$ and $\lim _{n \rightarrow \infty}\left\|\frac{x_{n}+y_{n}}{2}\right\|=1$. Let $U_{E}=\{x \in E:\|x\|=1\}$ be the unit sphere of $E$. Then the Banach space $E$ is said to be smooth provided

$$
\lim _{t \rightarrow 0} \frac{\|x+t y\|-\|x\|}{t}
$$

exists for all $x, y \in U_{E}$. It is also said to be uniformly smooth if the limit (1.1) is attained uniformly for all $x, y \in U_{E}$. It is well known that if $E$ is uniformly smooth, then $J$ is uniformly norm-to-norm continuous on each bounded subset of $E$. It is also well known that if $E$ is uniformly smooth if and only if $E^{*}$ is uniformly convex.

Let $\rightarrow$ and $\rightarrow$ denote the weak and strong convergence, respectively. Recall that a Banach space $E$ has the Kadec-Klee property if for any sequence $\left\{x_{n}\right\} \subset$ $E$ and $x \in E$ with $x_{n} \rightarrow x$ and $\left\|x_{n}\right\| \rightarrow\|x\|$, then $\left\|x_{n}-x\right\| \rightarrow 0$ as $n \rightarrow \infty$ for more details on Kadec-Klee property, the readers is referred to [7,27] and the references therein. It is well known that if $E$ is a uniformly convex Banach spaces, then $E$ enjoys the Kadec-Klee property.

Let $C$ be a nonempty closed and convex subset of a Banach space $E$, and $T: C \rightarrow C$ a mapping. The mapping $T$ is said to be closed if for any sequence $\left\{x_{n}\right\} \subset C$ such that $\lim _{n \rightarrow \infty} x_{n}=x_{0}$ and $\lim _{n \rightarrow \infty} T x_{n}=y_{0}$, then $T x_{0}=y_{0}$. The mapping $T$ is said to be asymptotically regular on $C$ if

$$
\lim _{n \rightarrow \infty} \sup _{x \in C}\left\|T^{n+1} x-T^{n} x\right\|=0 .
$$

A point $x \in C$ is a fixed point of $T$ provided $T x=x$. In this paper, we use $F(T)$ to denote the fixed point set of $T$.

As we all know that if $C$ is a nonempty closed convex subset of a Hilbert space $H$, and $P_{C}: H \rightarrow C$ is the metric projection of $H$ onto $C$, then $P_{C}$ is nonexpansive. This fact actually characterizes Hilbert spaces and consequently, it is not available in more general Banach spaces. In this connection, Alber [3] recently introduced a generalized projection operator $\Pi_{C}$ in a Banach space $E$ which is an analogue of the metric projection in Hilbert spaces.

Next, we assume that $E$ is a smooth Banach space. Consider the functional defined by

$$
\phi(x, y)=\|x\|^{2}-2\langle x, J y\rangle+\|y\|^{2} \quad \text { for } x, y \in E .
$$

Observe that, in a Hilbert space $H,(1.2)$ is reduced to $\phi(x, y)=\|x-y\|^{2}$, $x, y \in H$. The generalized projection $\Pi_{C}: E \rightarrow C$ is a map that assigns to an arbitrary point $x \in E$ the minimum point of the functional $\phi(x, y)$, that is, 
$\Pi_{C} x=\bar{x}$, where $\bar{x}$ is the solution to the minimization problem

$$
\phi(\bar{x}, x)=\min _{y \in C} \phi(y, x) .
$$

Existence and uniqueness of the operator $\Pi_{C}$ follows from the properties of the functional $\phi(x, y)$ and strict monotonicity of the mapping $J$ (see, for example, $[2,3,7,27])$. In Hilbert spaces, $\Pi_{C}=P_{C}$. It is obvious from the definition of function $\phi$ that

$$
(\|y\|-\|x\|)^{2} \leq \phi(y, x) \leq(\|y\|+\|x\|)^{2}, \quad \forall x, y \in E .
$$

Remark 1.1. If $E$ is a reflexive, strictly convex and smooth Banach space, then for $x, y \in E, \phi(x, y)=0$ if and only if $x=y$. It is sufficient to show that if $\phi(x, y)=0$ then $x=y$. From (1.3), we have $\|x\|=\|y\|$. This implies that $\langle x, J y\rangle=\|x\|^{2}=\|J y\|^{2}$. From the definition of $J$, we have $J x=J y$. Therefore, we have $x=y$; see $[7,27]$ for more details.

Let $C$ be a nonempty closed convex subset of $E$, and $T$ a mapping from $C$ into itself. A point $p$ in $C$ is said to be an asymptotic fixed point of $T$ [25] if $C$ contains a sequence $\left\{x_{n}\right\}$ which converges weakly to $p$ such that $\lim _{n \rightarrow \infty}\left\|x_{n}-T x_{n}\right\|=0$. The set of asymptotic fixed points of $T$ will be denoted by $\widetilde{F}(T)$.

$T$ is said to be nonexpansive if

$$
\|T x-T y\| \leq\|x-y\|, \quad \forall x, y \in C .
$$

It is well known that if $C$ is a nonempty, bounded, closed and convex subset of a uniformly convex Banach space $E$, then every nonexpansive self-mapping $T$ on $C$ has a fixed point. Further, the fixed point set of $T$ is closed and convex.

$T$ is said to be quasi-nonexpansive if $F(T) \neq \emptyset$ and

$$
\|p-T x\| \leq\|p-x\|, \quad \forall p \in F(T), x \in C .
$$

$T$ is said to be relatively nonexpansive [2-4] if $\widetilde{F}(T)=F(T) \neq \emptyset$ and

$$
\phi(p, T x) \leq \phi(p, x), \quad \forall p \in F(T), x \in C .
$$

The asymptotic behavior of relatively nonexpansive mappings was studied in [4-6]. There are several convergence theorems for fixed points of relatively nonexpansive mappings were established, see, for example, [11,17,24,28].

$T$ is said to be $\phi$-nonexpansive $[18,21,30]$ if

$$
\phi(T x, T y) \leq \phi(x, y), \quad \forall x, y \in C .
$$


Remark 1.2. In Hilbert spaces, the class of $\phi$-nonexpansive mappings is reduced to the class of nonexpansive mappings.

$T$ is said to be quasi- $\phi$-nonexpansive $[15,18,24]$ if $F(T) \neq \emptyset$ and

$$
\phi(p, T x) \leq \phi(p, x), \quad \forall p \in F(T), x \in C .
$$

Remark 1.3. The class of quasi- $\phi$-nonexpansive mappings is more general than the class of relatively nonexpansive mappings which requires the restriction: $F(T)=\widetilde{F}(T)$.

Remark 1.4. In Hilbert spaces, the class of quasi- $\phi$-nonexpansive mappings is reduced to the class of quasi-nonexpansive mappings.

$T$ is said to be asymptotically nonexpansive if there exists a sequence $\left\{k_{n}\right\} \subset[1, \infty)$ with $k_{n} \rightarrow 1$ as $n \rightarrow \infty$ such that

$$
\left\|T^{n} x-T^{n} y\right\| \leq k_{n}\|x-y\|, \quad \forall x, y \in C, \forall n \geq 1 .
$$

The class of asymptotically nonexpansive mappings was introduced by Goebel and Kirk [13] in 1972. Since 1972, a host of authors have studied the weak and strong convergence of iterative processes for such a class of mappings.

$T$ is said to be asymptotically quasi-nonexpansive if $F(T) \neq \emptyset$ and there exists a sequence $\left\{k_{n}\right\} \subset[1, \infty)$ with $k_{n} \rightarrow 1$ as $n \rightarrow \infty$ such that

$$
\left\|p-T^{n} x\right\| \leq k_{n}\|p-x\|, \quad \forall p \in F(T), x \in C, \forall n \geq 1
$$

$T$ is said to be relatively asymptotically nonexpansive [1,23] if $\widetilde{F}(T)=$ $F(T) \neq \emptyset$ and there exists a sequence $\left\{k_{n}\right\} \subset[1, \infty)$ with $k_{n} \rightarrow 1$ as $n \rightarrow \infty$ such that

$$
\phi\left(p, T^{n} x\right) \leq k_{n} \phi(p, x), \quad \forall p \in F(T), x \in C, \forall n \geq 1 .
$$

$T$ is said to be asymptotically $\phi$-nonexpansive $[22,30]$ if there exists a sequence $\left\{k_{n}\right\} \subset[1, \infty)$ with $k_{n} \rightarrow 1$ as $n \rightarrow \infty$ such that

$$
\phi\left(T^{n} x, T^{n} y\right) \leq k_{n} \phi(x, y), \quad \forall x, y \in C, \forall n \geq 1 .
$$

Remark 1.5. In Hilbert spaces, the class of asymptotically $\phi$-nonexpansive mappings is reduced to the class of asymptotically nonexpansive mappings.

$T$ is said to be asymptotically quasi- $\phi$-nonexpansive $[19,22,30]$ if $F(T) \neq \emptyset$ and there exists a sequence $\left\{k_{n}\right\} \subset[1, \infty)$ with $k_{n} \rightarrow 1$ as $n \rightarrow \infty$ such that

$$
\phi\left(p, T^{n} x\right) \leq k_{n} \phi(p, x), \quad \forall p \in F(T), x \in C, \forall n \geq 1 .
$$


Remark 1.6. The class of asymptotically quasi- $\phi$-nonexpansive mappings is more general than the class of relatively asymptotically nonexpansive mappings which requires the restriction: $F(T)=\widetilde{F}(T)$.

Remark 1.7. In Hilbert spaces, the class of asymptotically quasi- $\phi$-nonexpansive mappings is reduced to the class of asymptotically quasi-nonexpansive mappings.

In 1976, Halpern [15] introduced the following explicit iteration for a nonexpansive mapping:

$$
x_{0} \in C, \quad x_{n+1}=\alpha_{n} u+\left(1-\alpha_{n}\right) T x_{n}, \quad \forall n \geq 0 .
$$

He pointed out that the conditions

(C1) $\lim _{n \rightarrow \infty} \alpha_{n}=0$;

(C2) $\sum_{n=1}^{\infty} \alpha_{n}=\infty$

are necessary in the sense that if the iteration converges to a fixed point of $T$, then these conditions must be satisfied. It is well know that the process is widely believed to have slow convergence because the restriction of the condition (C2). To improve the rate of convergence of Halpern iteration, one cannot rely only on the process itself. The hybrid projection method which was first considered by Hangazeau [11] has been employed to study Halpern iteration and other mean iterations by many authors; see, for example, [8$12,16-23,26,29]$.

In 2007, Qin and Su [20] considered modifying Halpern iteration for a relatively nonexpansive mapping. To be more precise, they obtained the following results.

Theorem QS. Let $E$ be a uniformly convex and uniformly smooth Banach space, let $C$ be a nonempty closed convex subset of $E$, let $T: C \rightarrow C$ be a relatively nonexpansive mapping. Assume that $\left\{\alpha_{n}\right\}$ is a sequence in $(0,1)$ such that $\lim _{n \rightarrow \infty} \alpha_{n}=0$. Define a sequence $\left\{x_{n}\right\}$ in $C$ by the following algorithm:

$$
\left\{\begin{array}{l}
x_{0} \in C \quad \text { chosen arbitrarily, } \\
y_{n}=J^{-1}\left(\alpha_{n} J x_{0}+\left(1-\alpha_{n}\right) J T x_{n}\right), \\
C_{n}=\left\{v \in C: \phi\left(v, y_{n}\right) \leq \alpha_{n} \phi\left(v, x_{0}\right)+\left(1-\alpha_{n}\right) \phi\left(v, x_{n}\right),\right. \\
Q_{n}=\left\{v \in C:\left\langle J x_{0}-J x_{n}, x_{n}-v\right\rangle \geq 0\right\} \\
x_{n+1}=\Pi_{C_{n} \cap Q_{n}} x_{0}, \quad \forall n \geq 0
\end{array}\right.
$$

where $J$ is the single-valued duality mapping on $E$. If $F(T)$ is nonempty, then $\left\{x_{n}\right\}$ converges to $\Pi_{F(T)} x_{0}$. 
Recently, Qin, Cho, Kang and Zhou [18] further improved Theorem QS by considering quasi- $\phi$-nonexpansive mappings. To be more precise, they proved the following.

Theorem QCKZ. Let $C$ be a nonempty closed convex subset of a uniformly convex and uniformly smooth Banach space $E$ and $T: C \rightarrow C$ a closed and quasi- $\phi$-nonexpansive mapping such that $F(T) \neq \emptyset$. Let $\left\{x_{n}\right\}$ be a sequence generated in the following manner:

$$
\left\{\begin{array}{l}
x_{0} \in E \quad \text { chosen arbitrarily, } \\
C_{1}=C \\
x_{1}=\Pi_{C_{1}} x_{0} \\
y_{n}=J^{-1}\left(\alpha_{n} J x_{1}+\left(1-\alpha_{n}\right) J T x_{n}\right) \\
C_{n+1}=\left\{z \in C_{n}: \phi\left(z, y_{n}\right) \leq \alpha_{n} \phi\left(z, x_{1}\right)+\left(1-\alpha_{n}\right) \phi\left(z, x_{n}\right)\right\} \\
x_{n+1}=\Pi_{C_{n+1}} x_{1} .
\end{array}\right.
$$

Assume that the control sequence satisfies the restriction: $\lim _{n \rightarrow \infty} \alpha_{n}=0$. Then $\left\{x_{n}\right\}$ converges strongly to $\Pi_{F(T)} x_{1}$.

Very recently, Cho, Qin and Kang [12] reconsidered Halpern iteration for an asymptotically quasi- $\phi$-nonexpansive mapping. To be more precise, they proved the following.

Theorem CQK. Let $C$ be a nonempty closed convex subset of a uniformly convex and uniformly smooth Banach space $E$ and $T: C \rightarrow C$ a closed asymptotically quasi- $\phi$-nonexpansive mapping with a sequence $\left\{k_{n}\right\} \subset[1, \infty)$ such that $k_{n} \rightarrow 1$ as $n \rightarrow \infty$. Assume that $T$ is asymptotically regular on $C$, $F(T) \neq \emptyset$ and $F(T)$ is bounded. Let $\left\{x_{n}\right\}$ be a sequence generated in the following manner:

$$
\left\{\begin{array}{l}
x_{0} \in E \text { chosen arbitrarily, } \\
C_{1}=C \\
x_{1}=\Pi_{C_{1}} x_{0} \\
y_{n}=J^{-1}\left[\alpha_{n} J x_{1}+\left(1-\alpha_{n}\right) J T^{n} x_{n}\right] \\
C_{n+1}=\left\{z \in C_{n}: \phi\left(z, y_{n}\right) \leq \phi\left(z, x_{n}\right)+\alpha_{n} M\right\} \\
x_{n+1}=\Pi_{C_{n+1}} x_{1}, \quad \forall n \geq 0
\end{array}\right.
$$

where $M$ is an appropriate constant such that $M \geq \phi\left(w, x_{1}\right)$ for all $w \in$ $F(T)$. Assume that the control sequence $\left\{\alpha_{n}\right\}$ in $(0,1)$ satisfies the following restrictions:

(a) $\lim _{n \rightarrow \infty} \alpha_{n}=0$,

(b) $\left(1-\alpha_{n}\right) k_{n} \leq 1$ for all $n \geq 0$. 
Then $\left\{x_{n}\right\}$ converges strongly to $\Pi_{F(T)} x_{1}$.

In this paper, motivated by the research going on in this direction, we reconsider modifying Halpern iteration based on hybrid projection methods for a family of asymptotically quasi- $\phi$-nonexpansive mappings. Strong convergence theorems are established in a uniformly smooth and strictly convex Banach space which also enjoys the Kadec-Klee property. Note that every uniformly convex Banach space enjoys the Kadec-Klee property. The results presented in this paper can be viewed as an improvement of Cho, Qin and Kang [12], Martinez-Yanes Xu [16], Qin, Cho, Kang and Zhou [18], Qin and Su [20].

In order to our main results, we need the following lemmas.

Lemma 1.1 ([3]). Let $C$ be a nonempty closed convex subset of a smooth Banach space $E$ and $x \in E$. Then, $x_{0}=\Pi_{C} x$ if and only if

$$
\left\langle x_{0}-y, J x-J x_{0}\right\rangle \geq 0 \quad \forall y \in C
$$

Lemma 1.2. ([3]). Let $E$ be a reflexive, strictly convex and smooth Banach space, $C$ a nonempty closed convex subset of $E$ and $x \in E$. Then

$$
\phi\left(y, \Pi_{C} x\right)+\phi\left(\Pi_{C} x, x\right) \leq \phi(y, x) \quad \forall y \in C .
$$

Lemma 1.3. ([19]). Let $E$ be a uniformly smooth and strictly convex Banach space which also enjoys the Kadec-Klee property, $C$ a nonempty closed convex subset of $E$ and $T: C \rightarrow C$ a closed and asymptotically quasi- $\phi$-nonexpansive mapping. Then $F(T)$ is a closed convex subset of $C$.

\section{Main results}

Theorem 2.1. Let $E$ be a uniformly smooth and strictly convex Banach space which also enjoys the Kadec-Klee property, and $C$ a nonempty closed convex subset of $E$. Let $T_{i}: C \rightarrow C$ be an asymptotically regular, closed and asymptotically quasi- $\phi$-nonexpansive mapping with the sequences $\left\{k_{n, i}\right\} \subset$ $\left[1, \frac{1}{1-\alpha_{n, i}}\right]$ for each $i \geq 1$. Assume that $F\left(T_{i}\right)$ is bounded for each $i \geq 1$, and $\mathcal{F}=\cap_{i=1}^{\infty} F\left(T_{i}\right)$ is nonempty. Let $\left\{x_{n}\right\}$ be a sequence generated in the following 
manner:

$$
\left\{\begin{array}{l}
x_{0} \in E \quad \text { chosen arbitrarily, } \\
C_{1, i}=C, \quad C_{1}=\cap_{i=1}^{\infty} C_{1, i} \\
x_{1}=\Pi_{C_{1}} x_{0} \\
y_{n, i}=J^{-1}\left(\alpha_{n, i} J x_{1}+\left(1-\alpha_{n, i}\right) J T_{i}^{n} x_{n}\right), \quad n \geq 1, \\
C_{n+1, i}=\left\{z \in C_{n, i}: \phi\left(z, y_{n, i}\right) \leq \phi\left(z, x_{n}\right)+\alpha_{n, i} M\right\} \\
C_{n+1}=\cap_{i=1}^{\infty} C_{n+1, i} \\
x_{n+1}=\Pi_{C_{n+1}} x_{1}, \forall n \geq 0
\end{array}\right.
$$

where $M=\sup \left\{\phi\left(z, x_{1}\right): z \in \mathcal{F}\right\}$. Assume that the control sequence $\left\{\alpha_{n, i}\right\}$ is chosen such that $\lim _{n \rightarrow \infty} \alpha_{n, i}=0$ for each $i \geq 1$. Then the sequence $\left\{x_{n}\right\}$ converges strongly to $\Pi_{\mathcal{F}} x_{1}$.

Proof. First, we show that $C_{n}$ is closed and convex for each $n \geq 1$. It suffices to claim that, $\forall i \geq 1, C_{n, i}$ is closed and convex for every $n \geq 1$. This can be proved by induction on $n$. In fact, for $n=1, C_{1, i}=C$ is closed and convex. Assume that $C_{h, i}$ is closed and convex for some $h$. For $z \in C_{h, i}$, we see that $\phi\left(z, y_{h, i}\right) \leq \phi\left(z, x_{h}\right)+\alpha_{h, i} M$ is equivalent to

$$
2\left\langle z, J x_{h}-J y_{h, i}\right\rangle \leq\left\|x_{h}\right\|^{2}-\left\|y_{h, i}\right\|^{2}+\alpha_{h, i} M .
$$

Hence $C_{h+1, i}$ is closed and convex for each $i$. Hence, for $\forall i \geq 1 C_{n, i}$ is closed and convex. This proves that $C_{n}$ is closed and convex for each $n \geq 1$.

Next, we prove that $\mathcal{F} \subset C_{n}$ for each $n \geq 1$. It suffices to claim that $\mathcal{F} \subset C_{n, i}$ for each $n \geq 1$ and for each $i \geq 1$. Note that $\mathcal{F} \subset C_{1, i}=C$. Suppose that $\mathcal{F} \subset C_{h, i}$ for some $h$ and for all $i$. Then, for $\forall w \in \mathcal{F} \subset C_{h, i}$, we have

$$
\begin{aligned}
& \phi\left(w, y_{h, i}\right) \\
& =\phi\left(w, J^{-1}\left(\alpha_{h, i} J x_{1}+\left(1-\alpha_{h, i}\right) J T_{i}^{h} x_{h}\right)\right) \\
& =\|w\|^{2}-2\left\langle w, \alpha_{h, i} J x_{1}+\left(1-\alpha_{h, i}\right) J T_{i}^{h} x_{h}\right\rangle+\left\|\alpha_{h, i} J x_{1}+\left(1-\alpha_{h, i}\right) J T_{i}^{h} x_{h}\right\|^{2} \\
& \leq\|w\|^{2}-2 \alpha_{h, i}\left\langle w, J x_{1}\right\rangle-2\left(1-\alpha_{h, i}\right)\left\langle w, J T_{i}^{h} x_{h}\right\rangle+\alpha_{h, i}\left\|x_{1}\right\|^{2}+\left(1-\alpha_{h, i}\right)\left\|T_{i}^{h} x_{h}\right\|^{2} \\
& =\alpha_{h, i} \phi\left(w, x_{1}\right)+\left(1-\alpha_{h, i}\right) \phi\left(w, T_{i}^{h} x_{h}\right) \\
& \leq \alpha_{h, i} \phi\left(w, x_{1}\right)+\left(1-\alpha_{h, i}\right) k_{h, i} \phi\left(w, x_{h}\right) \\
& =\phi\left(w, x_{h}\right)-\left(1-\left(1-\alpha_{h, i}\right) k_{h, i}\right) \phi\left(w, x_{h}\right)+\alpha_{h, i} \phi\left(w, x_{1}\right) \\
& \leq \phi\left(w, x_{h}\right)+\alpha_{h, i} \phi\left(w, x_{1}\right) \\
& \leq \phi\left(w, x_{h}\right)+\alpha_{h, i} M
\end{aligned}
$$

which shows that $w \in C_{h+1, i}$. This implies that $\mathcal{F} \subset C_{n, i}$ for each $n \geq 1$ and each $i \geq 1$. This proves that $\mathcal{F} \subset C_{n}$ for each $n \geq 1$.

On the other hand, we see from Lemma 1.2 that

$$
\phi\left(x_{n}, x_{1}\right)=\phi\left(\Pi_{C_{n}} x_{1}, x_{1}\right) \leq \phi\left(w, x_{1}\right)-\phi\left(w, x_{n}\right) \leq \phi\left(w, x_{1}\right)
$$


for each $w \in \mathcal{F} \subset C_{n}$ and for each $n \geq 1$. This shows that the sequence $\phi\left(x_{n}, x_{1}\right)$ is bounded. From (1.3), we see that the sequence $\left\{x_{n}\right\}$ is also bounded. Since the space is reflexive, we may, without loss of generality, assume that $x_{n} \rightarrow p$. Since $C_{m} \subset C_{n}$ for all $m \geq n$, we have $x_{m} \in C_{n}$ for all $m \geq n$. Since $C_{n}$ is closed and convex, we see that $p \in C_{n}$ for all $n \geq 1$. It follows that $p \in \cap_{n=1}^{\infty} C_{n}$. In view of $\phi\left(x_{n}, x_{1}\right) \leq \phi\left(x_{n+1}, x_{1}\right) \leq \phi\left(p, x_{1}\right)$, we see that

$$
\phi\left(p, x_{1}\right) \leq \liminf _{n \rightarrow \infty} \phi\left(x_{n}, x_{1}\right) \leq \limsup _{n \rightarrow \infty} \phi\left(x_{n}, x_{1}\right) \leq \phi\left(p, x_{1}\right) .
$$

This implies that

$$
\lim _{n \rightarrow \infty} \phi\left(x_{n}, x_{1}\right)=\phi\left(p, x_{1}\right) .
$$

Hence, we have $\left\|x_{n}\right\| \rightarrow\|p\|$ as $n \rightarrow \infty$. In view of the Kadec-Klee property of $E$, we obtain that

$$
\lim _{n \rightarrow \infty} x_{n}=p .
$$

Next, we show that $p \in \mathcal{F}$. By the construction of $C_{n}$, we have that $C_{n+1} \subset C_{n}$ and $x_{n+1}=\Pi_{C_{n+1}} x_{1} \in C_{n}$. It follows that

$$
\begin{aligned}
\phi\left(x_{n+1}, x_{n}\right) & =\phi\left(x_{n+1}, \Pi_{C_{n}} x_{1}\right) \\
& \leq \phi\left(x_{n+1}, x_{1}\right)-\phi\left(\Pi_{C_{n}} x_{1}, x_{1}\right) \\
& =\phi\left(x_{n+1}, x_{1}\right)-\phi\left(x_{n}, x_{1}\right) .
\end{aligned}
$$

Letting $n \rightarrow \infty$, we obtain that $\phi\left(x_{n+1}, x_{n}\right) \rightarrow 0$. In view of $x_{n+1} \in C_{n+1}$, we obtain that

$$
\phi\left(x_{n+1}, y_{n, i}\right) \leq \phi\left(x_{n+1}, x_{n}\right)+\alpha_{n, i} M, \quad \forall i \geq 1
$$

It follows that

$$
\lim _{n \rightarrow \infty} \phi\left(x_{n+1}, y_{n, i}\right)=0, \quad \forall i \geq 1 .
$$

From (1.3), we see that

$$
\lim _{n \rightarrow \infty}\left\|y_{n, i}\right\|=\|p\|, \quad \forall i \geq 1
$$

It follows that

$$
\lim _{n \rightarrow \infty}\left\|J y_{n, i}\right\|=\|J p\|, \quad \forall i \geq 1 .
$$

This implies that $\left\{J y_{n, i}\right\}$ is bounded. Note that $E$ is reflexive and $E^{*}$ is also reflexive. We may assume that $J y_{n, i} \rightarrow x^{i, *} \in E^{*}$ for each $i \geq 1$. In view of the reflexivity of $E$, we see that $J(E)=E^{*}$. This shows that there exists an $x^{i} \in E$ such that $J x^{i}=x^{i, *}$. It follows that

$$
\begin{aligned}
\phi\left(x_{n+1}, y_{n, i}\right) & =\left\|x_{n+1}\right\|^{2}-2\left\langle x_{n+1}, J y_{n, i}\right\rangle+\left\|y_{n, i}\right\|^{2} \\
& =\left\|x_{n+1}\right\|^{2}-2\left\langle x_{n+1}, J y_{n, i}\right\rangle+\left\|J y_{n, i}\right\|^{2} .
\end{aligned}
$$


Taking $\liminf \operatorname{in}_{n \rightarrow \infty}$ the both sides of equality above yields that

$$
\begin{aligned}
0 & \geq\|p\|^{2}-2\left\langle p, x^{i, *}\right\rangle+\left\|x^{i, *}\right\|^{2} \\
& =\|p\|^{2}-2\left\langle p, J x^{i}\right\rangle+\left\|J x^{i}\right\|^{2} \\
& =\|p\|^{2}-2\left\langle p, J x^{i}\right\rangle+\left\|x^{i}\right\|^{2} \\
& =\phi\left(p, x^{i}\right), \quad \forall i \geq 1 .
\end{aligned}
$$

That is, $p=x^{i}$, which in turn implies that $x^{i, *}=J p$ for each $i \geq 1$. It follows that $J y_{n, i} \rightarrow J p \in E^{*}$ for each $i \geq 1$. Since (2.4) and $E^{*}$ enjoys the Kadec-Klee property, we obtain that

$$
\lim _{n \rightarrow \infty} J y_{n, i}=J p, \quad \forall i \geq 1 .
$$

Note that $J^{-1}: E^{*} \rightarrow E$ is demi-continuous. It follows that $y_{n, i} \rightarrow p$ for each $i \geq 1$. Since (2.3) and $E$ enjoys the Kadec-Klee property, we obtain that

$$
\lim _{n \rightarrow \infty} y_{n, i}=p, \quad \forall i \geq 1 .
$$

Note that

$$
\left\|x_{n}-y_{n, i}\right\| \leq\left\|x_{n}-p\right\|+\left\|p-y_{n, i}\right\|, \quad \forall 1 \geq 1 .
$$

It follows that

$$
\lim _{n \rightarrow \infty}\left\|x_{n}-y_{n, i}\right\|=0, \quad \forall i \geq 1 .
$$

Since $J$ is uniformly norm-to-norm continuous on any bounded sets, we have

$$
\lim _{n \rightarrow \infty}\left\|J x_{n}-J y_{n, i}\right\|=0, \quad \forall i \geq 1 .
$$

Notice from (2.1) that

$$
J x_{n}-J y_{n, i}=\alpha_{n, i}\left(J x_{n}-J x_{1}\right)+\left(1-\alpha_{n, i}\right)\left(J x_{n}-J T_{i}^{n} x_{n}\right)
$$

It follows that

$$
\lim _{n \rightarrow \infty}\left\|J x_{n}-J T_{i}^{n} x_{n}\right\|=0, \quad \forall i \geq 1 .
$$

Since $J$ is uniformly norm-to-norm continuous on any bounded sets, we see from (2.2) that

$$
\lim _{n \rightarrow \infty}\left\|J x_{n}-J p\right\|=0 .
$$

Notice that

$$
\left\|J T_{i}^{n} x_{n}-J p\right\| \leq\left\|J T_{i}^{n} x_{n}-J x_{n}\right\|+\left\|J x_{n}-J p\right\|, \quad \forall i \geq 1 .
$$

In view of (2.8) and (2.9), we have

$$
\lim _{n \rightarrow \infty}\left\|J T_{i}^{n} x_{n}-J p\right\|=0 .
$$


The demi-continuity of $J^{-1}: E^{*} \rightarrow E$ implies that $T_{i}^{n} x_{n} \rightarrow p$ for each $i$. Note that

$$
\left|\left\|T_{i}^{n} x_{n}\right\|-\|p\|\right|=\left|\left\|J T_{i}^{n} x_{n}\right\|-\|J p\|\right| \leq\left\|J T_{i}^{n} x_{n}-J p\right\|, \quad \forall i \geq 1 .
$$

From (2.10), we see that $\left\|T_{i}^{n} x_{n}\right\| \rightarrow\|p\|$, for each $i \geq 1$, as $n \rightarrow \infty$. Since $E$ has the Kadec-Klee property, we obtain that

$$
\lim _{n \rightarrow \infty}\left\|T_{i}^{n} x_{n}-p\right\|=0, \quad \forall i \geq 1
$$

Since

$$
\left\|T_{i}^{n+1} x_{n}-p\right\| \leq\left\|T_{i}^{n+1} x_{n}-T_{i}^{n} x_{n}\right\|+\left\|T_{i}^{n} x_{n}-p\right\|, \quad \forall i \geq 1 .
$$

It follows from the asymptotic regularity of $T_{i}$ and (2.11) that

$$
\lim _{n \rightarrow \infty}\left\|T_{i}^{n+1} x_{n}-p\right\|=0,
$$

that is, $T_{i} T_{i}^{n} x_{n}-p \rightarrow 0$ as $n \rightarrow \infty$. It follows from the closedness of $T_{i}$ that $T_{i} p=p$ for each $i \geq 1$. This proves that $p \in \mathcal{F}$.

Finally, we show that $p=\Pi_{\mathcal{F}} x_{1}$. From $x_{n}=\Pi_{C_{n}} x_{1}$, we have

$$
\left\langle x_{n}-w, J x_{1}-J x_{n}\right\rangle \geq 0, \quad \forall w \in \mathcal{F} \subset C_{n} .
$$

Taking the limit as $n \rightarrow \infty$ in (2.12), we obtain that

$$
\left\langle p-w, J x_{1}-J p\right\rangle \geq 0, \quad \forall w \in \mathcal{F} .
$$

In view of Lemma 1.1 , we see that $p=\Pi_{\mathcal{F}} x_{1}$. This completes the proof.

Remark 2.2. Note that every uniformly convex Banach space enjoys the Kadec-Klee property. Theorem 2.1 improves Theorem CQK in following aspects:

(a) from the viewpoint of the space, the space is extended from uniformly smooth and uniformly convex Banach spaces to uniformly smooth and strictly convex Banach spaces which enjoy the Kadec-Klee property;

(b) from the viewpoint of the mapping, a family of mappings is considered instead of a single mapping.

For the class of quasi- $\phi$-nonexpansive mappings, we have from Theorem 2.1 the following immediately.

Corollary 2.3. Let $E$ be a uniformly smooth and strictly convex Banach space which also enjoys the Kadec-Klee property and $C$ a nonempty closed 
convex subset of $E$. Let $T_{i}: C \rightarrow C$ be a closed quasi- $\phi$-nonexpansive mapping such that $F\left(T_{i}\right) \neq \emptyset$ for each $i \geq 1$. Let $\left\{x_{n}\right\}$ be a sequence generated in the following manner:

$$
\left\{\begin{array}{l}
x_{0} \in E \quad \text { chosen arbitrarily, } \\
C_{1, i}=C, \quad C_{1}=\cap_{i=1}^{\infty} C_{1, i}, \\
x_{1}=\Pi_{C_{1}} x_{0} \\
y_{n, i}=J^{-1}\left(\alpha_{n, i} J x_{1}+\left(1-\alpha_{n, i}\right) J T_{i} x_{n}\right), \quad n \geq 1, \\
C_{n+1, i}=\left\{z \in C_{n, i}: \phi\left(z, y_{n, i}\right) \leq \alpha_{n, i} \phi\left(z, x_{1}\right)+\left(1-\alpha_{n, i}\right) \phi\left(z, x_{n}\right)\right\} \\
C_{n+1}=\cap_{i=1}^{\infty} C_{n+1, i}, \\
x_{n+1}=\Pi_{C_{n+1}} x_{1}, \forall n \geq 0 .
\end{array}\right.
$$

Assume that the control sequence $\left\{\alpha_{n, i}\right\}$ is chosen such that $\lim _{n \rightarrow \infty} \alpha_{n, i}=0$ for each $i \geq 1$. Then the sequence $\left\{x_{n}\right\}$ converges strongly to $\Pi_{\mathcal{F}} x_{1}$.

In Hilbert spaces, Corollary 2.3 is reduced to the following immediately.

Corollary 2.4. Let $H$ be a Hilbert space and $C$ a nonempty closed convex subset of $H$. Let $T_{i}: C \rightarrow C$ be a closed quasi-nonexpansive mapping such that $F\left(T_{i}\right) \neq \emptyset$ for each $i \geq 1$. Let $\left\{x_{n}\right\}$ be a sequence generated in the following manner:

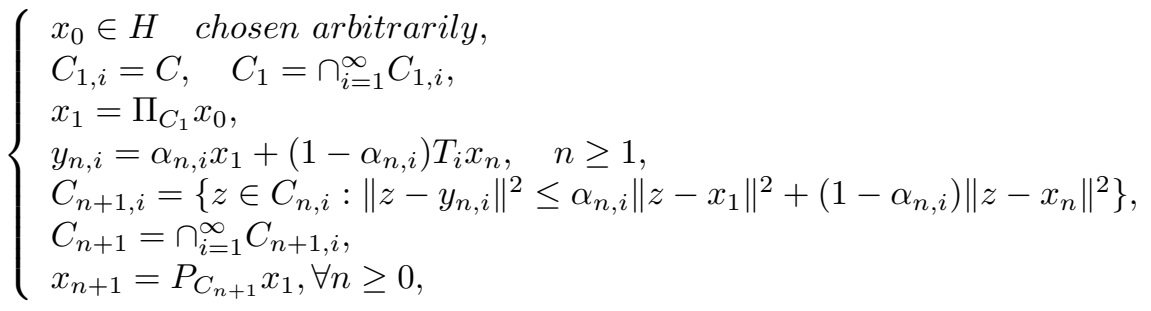

where $P$ is the metric projection. Assume that the control sequence $\left\{\alpha_{n, i}\right\}$ is chosen such that $\lim _{n \rightarrow \infty} \alpha_{n, i}=0$ for each $i \geq 1$. Then the sequence $\left\{x_{n}\right\}$ converges strongly to $P_{\mathcal{F}} x_{1}$.

For a single mapping, we can also obtain from the Theorem 2.1 the following easily.

Corollary 2.5. Let $E$ be a uniformly smooth and strictly convex Banach space which also enjoys the Kadec-Klee property and $C$ a nonempty closed convex subset of $E$. Let $T: C \rightarrow C$ be an asymptotically regular, closed and asymptotically quasi- $\phi$-nonexpansive mapping with the sequence $\left\{k_{n}\right\} \subset$ $\left[1, \frac{1}{1-\alpha_{n}}\right]$. Assume that $F(T)$ is bounded and $F(T)$ is nonempty. Let $\left\{x_{n}\right\}$ be 
a sequence generated in the following manner:

$$
\left\{\begin{array}{l}
x_{0} \in E \quad \text { chosen arbitrarily, } \\
C_{1}=C, \\
x_{1}=\Pi_{C_{1}} x_{0}, \\
y_{n}=J^{-1}\left(\alpha_{n} J x_{1}+\left(1-\alpha_{n}\right) J T^{n} x_{n}\right), \quad n \geq 1, \\
C_{n+1}=\left\{z \in C_{n}: \phi\left(z, y_{n}\right) \leq \phi\left(z, x_{n}\right)+\alpha_{n} M\right\}, \\
x_{n+1}=\Pi_{C_{n+1}} x_{1}, \forall n \geq 0,
\end{array}\right.
$$

where $M=\sup \left\{\phi\left(z, x_{1}\right): z \in F(T)\right\}$. Assume that the control sequence $\left\{\alpha_{n}\right\}$ is chosen such that $\lim _{n \rightarrow \infty} \alpha_{n}=0$. Then the sequence $\left\{x_{n}\right\}$ converges strongly to $\Pi_{F(T)} x_{1}$.

Remark 2.6. If $T$ is closed quasi- $\phi$-nonexpansive, then Corollary 2.4 is a version of Theorem QCKZ in a uniformly smooth and strictly convex Banach space which also enjoys the Kadec-Klee property.

\section{Acknowledgments}

The authors thank the referees for their comments which led to improvement of the presentation of this paper.

The second author was supported by Natural Science Foundation of Zhejiang Province (Y6110270).

\section{References}

[1] R.P. Agarwal, Y.J. Cho, X. Qin, Generalized projection algorithms for nonlinear operators, Numer. Funct. Anal. Optim. 28 (2007) 1197-1215.

[2] Ya.I. Alber, S. Reich, An iterative method for solving a class of nonlinear operator equations in Banach spaces, Panamer. Math. J. 4 (1994) 39-54.

[3] Ya.I. Alber, Metric and generalized projection operators in Banach spaces: properties and applications, in: A.G. Kartsatos (Ed.), Theory and Applications of Nonlinear Operators of Accretive and Monotone Type (Marcel Dekker, New York, 1996).

[4] D. Butnariu, S. Reich, A.J. Zaslavski, Asymptotic behavior of relatively nonexpansive operators in Banach spaces, J. Appl. Anal. 7 (2001) 151174.

[5] D. Butnariu, S. Reich, A.J. Zaslavski, Weak convergence of orbits of nonlinear operators in reflexive Banach spaces, Numer. Funct. Anal. Optim. 24 (2003) 489-508. 
[6] Y. Censor, S. Reich, Iterations of paracontractions and firmly nonexpansive operatorswith applications to feasibility and optimization, Optim. 37 (1996) 323-339.

[7] I. Cioranescu, Geometry of Banach Spaces, Duality Mappings and Nonlinear Problems (Kluwer, Dordrecht, 1990).

[8] S.Y. Cho, X. Qin, S.M. Kang, Hybrid projection algorithms for treating common fixed points of a family of demicontinuous pseudocontractions, Appl. Math. Lett. 25 (2012) 854-857.

[9] S.Y. Cho, S.M. Kang, Approximation of fixed points of pseudocontraction semigroups based on a viscosity iterative process, Appl. Math. Lett. 24 (2011) 224-228.

[10] S.Y. Cho, S.M. Kang, On the convergence of iterative sequences for a family of nonexpansive mappings and inverse-strongly monotone mappings, An. Stiint. Univ. Ovidius Constanta Ser. Mat. 19 (2011) 49-66.

[11] G. Cai, H.S. Hu, On the strong convergence of the implicit iterative processes for a finite family of relatively weak quasi-nonexpansive mappings, Appl. Math. Lett. 23 (2010) 73-78.

[12] Y.J. Cho, X. Qin, S.M. Kang, Strong convergence of the modified Halpern-type iterative algorithms in Banach spaces, An. Stiint. Univ. Ovidius Constanta Ser. Mat. 17 (2009) 51-68.

[13] K. Goebel, W.A. Kirk, A fixed point theorem for asymptotically nonexpansive mappings. Proc. Am. Math. Soc. 35 (1972) 171-174.

[14] Y. Haugazeau, Sur les inéquations variationnelles et la minimisation de fonctionnelles convexes, Thése, Université de Paris, Paris, France (1968).

[15] B. Halpern, Fixed points of nonexpanding maps, Bull. Am. Math. Soc. 73 (1967) 957-961.

[16] C. Martinez-Yanes, H.K. Xu, Strong convergence of the CQ method for fixed point iteration processes, Nonlinear Anal. 64 (2006) 2400-2411.

[17] S. Plubtieng, K. Ungchittrakool, Strong convergence theorems for a common fixed point of two relatively nonexpansive mappings in a Banach space, J. Approx. Theory 149 (2007), 103-115.

[18] X. Qin, Y.J. Cho, S.M. Kang, H. Zhou, Convergence of a modified Halpern-type iteration algorithm for quasi- $\phi$-nonexpansive mappings, Appl. Math. Lett. 22 (2009) 1051-1055. 
[19] X. Qin, R.P. Agarwa, Shrinking projection methods for a pair of asymptotically quasi- $\phi$-nonexpansive mappings, Numer. Funct. Anal. Optim. 31 (2010) 1072-1089.

[20] X. Qin, Y. Su, Strong convergence theorem for relatively nonexpansive mappings in a Banach space, Nonlinear Anal. 67 (2007) 1958-1965.

[21] X. Qin, Y.J. Cho, S.M. Kang, Convergence theorems of common elements for equilibrium problems and fixed point problems in Banach spaces, J. Comput. Appl. Math. 225 (2009) 20-30.

[22] X. Qin, S.Y. Cho, S.M. Kang, On hybrid projection methods for asymptotically quasi- $\phi$-nonexpansive mappings, Appl. Math. Comput. 215 (2010) 3874-3883.

[23] X. Qin, Y. Su, C. Wu and K. Liu, Strong convergence theorems for nonlinear operators in Banach spacess, Comm. Appl. Nonlinear Anal. 14 (2007) 35-50.

[24] X. Qin, S.Y. Cho, S.M. Kang, Strong convergence of shrinking projection methods for quasi- $\phi$-nonexpansive mappings and equilibrium problems, J. Comput. Appl. Math. 234 (2010) 750-760.

[25] S. Reich, A weak convergence theorem for the alternating method with Bregman distance, in: A.G. Kartsatos (Ed.), Theory and Applicationsof Nonlinear Operators of Accretive and Monotone Type (Marcel Dekker, New York, 1996).

[26] Y. Su, Z. Wang, H. Xu, Strong convergence theorems for a common fixed point of two hemi-relatively nonexpansive mappings, Nonlinear Anal. 71 (2009) 5616-5628.

[27] W. Takahashi, Nonlinear Functional Analysis (Yokohama-Publishers, 2000).

[28] J. Ye, J. Huang, Strong convergence theorems for fixed point problems and generalized equilibrium problems of three relatively quasinonexpansive mappings in Banach spaces, J. Math. Comput. Sci. 1 (2011) 1-18.

[29] S. Yang, W. Li, Iterative solutions of a system of equilibrium problems in Hilbert spaces, Adv. Fixed Point Theory, 1 (2011) 15-26.

[30] H. Zhou, G. Gao, B. Tan, Convergence theorems of a modified hybrid algorithm for a family of quasi- $\phi$-asymptotically nonexpansive mappings, J. Appl. Math. Comput. 32 (2010) 453-464. 
Chang-Qun Wu,

School of Business and Administration,

Henan University,

Kaifeng 475000, China.

Email: kyls2003@yahoo.com.cn

Yan Hao,

School of Mathematics, Physics and Information Science,

Zhejiang Ocean University,

Zhoushan 316004, China.

Email: zjhaoyan@yahoo.cn 